\title{
Mid-Infrared Dual-Comb Spectroscopy for Real-Time Gas Analysis With an Optical Parametric Oscillator
}

\author{
Julien Mandon, Simona M. Cristescu, Frans J.M. Harren \\ Life Science Trace Gas Facility, Molecular and Laser Physics, Institute of Molecules and Materials, \\ Radboud University, P.O. Box 9010, 6500GL Nijmegen, The Netherlands \\ j.mandon@science.ru.nl
}

In the visible or near-infrared, dual-comb spectrometer based on mode-locked sources leads to high spectral resolution, low recording time and enhanced sensitivity with not traded off, since massively parallel broadband detection can be performed. One challenge is to transfer the benefit of dual-comb spectroscopy to the midinfrared where strong fundamental ro-vibrational transitions of various molecules and the large number of detectable molecules are of importance for gas analysis. The generation of coherent short-pulses in the midinfrared for dual-comb spectroscopy is achieved in different ways [1]. Among them, Optical parametric oscillators (OPOs) offer simultaneously high mid-infrared power and broadband spectral coverage [2]. Since 2012, few demonstrations of mid-infrared dual-comb spectrometers based on OPO have been reported [3,4]. In this paper, we present a dual-comb spectrometer working in the 3-5 $\mu \mathrm{m}$ spectral region for real-time gas analysis.

The setup consists of a light source based on an Optical Parametric Oscillator (OPO) with two outputs, descried previously in [4]. The outputs deliver up to $200 \mathrm{~mW}$, spectrally cover the infrared spectrum over $350 \mathrm{~nm}$ at once and can be tuned within seconds from 2.7 to $4.7 \mu \mathrm{m}\left(2130-3700 \mathrm{~cm}^{-1}\right)$. The first output beam probes the molecular gas sample placed into a Herriott type multipass absorption cell offering $37 \mathrm{~m}$ absorption pathlength. The output of the gas cell is then recombined with the second mid-infared beam from the OPO source onto a single point cooled fast photovoltaic detector. The beating signal is amplified, digitized, and Fourier transformed in real-time via a FPGA (Field-Programmable Gate Arrays) based data acquisition system. As the system stability does not allow coherent averaging, on-line averaging of multiple spectra is possible by using as a spectral reference a single absorption line. This reference line is used to re-calibrate each spectrum before averaging, greatly reducing the fluctuations caused by instabilities of the mid-infrared combs and improving the molecular absorption lineshape.

By detecting the heterodyned signal between the two mid-infrared beams, broadband spectra of molecular gases are recorded every $4 \mathrm{~ms}$ and a spectral resolution of $0.05 \mathrm{~cm}-1(1.5 \mathrm{GHz})$ is achieved. Figure 1a illustrates the capabilities of the spectrometer in the case of methane. In 220 second, the recorded spectrum has a signal to noise ratio of about 2400 leading to a minimal absorption coefficient of $1.1 \times 10-7 \mathrm{~cm}^{-1}$. The system stability is assessed by measuring the absorption of a single line of $100 \mathrm{ppm}$ methane over time. The system shows great stability as the measured absorption stays within $5 \%$ or $5 \mathrm{ppm}$. Figure $1 \mathrm{~b}$ shows the monitoring of more complex molecules such as acetone or ethanol, thanks to the broad spectral coverage of the light sources and the ability of the spectrometer for broadband spectral detection.
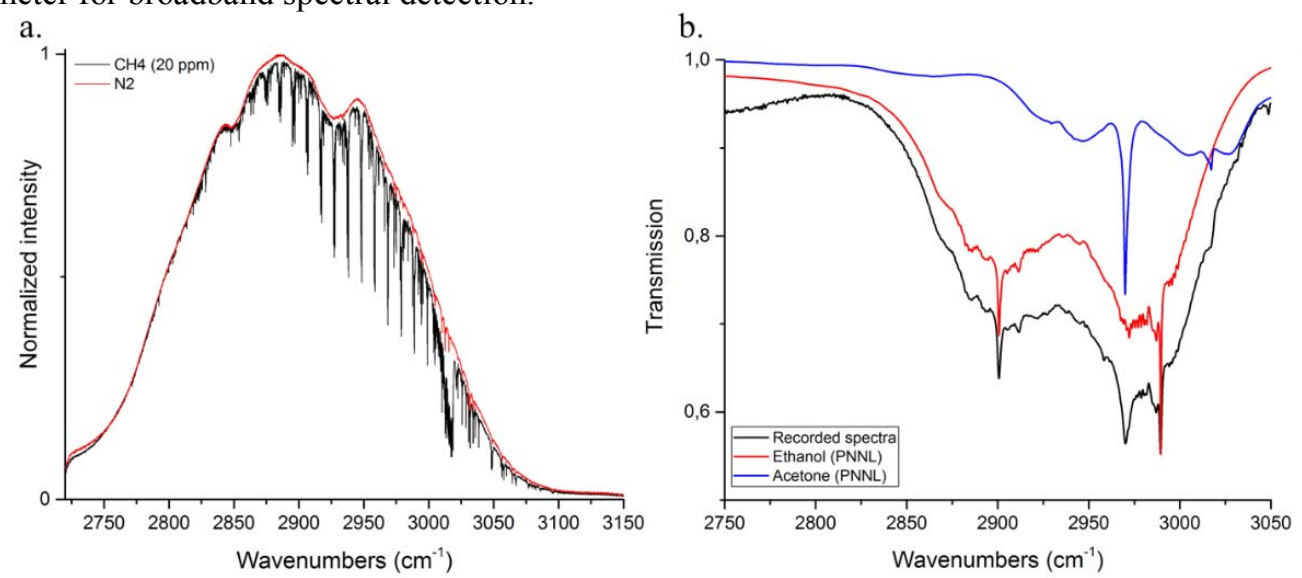

Fig. 1 Molecular spectra recorded with the Dual-comb based OPO setup. a/ Spectrum of 20 pppm methane. b/ Spectrum of a mixture of acetone and ethanol. The broadband light sources helps for the analysis of complex gas mixtures. When coupled with a multi-pass cell, the spectrometer allows ppm detection limits in few milliseconds.

\section{References}

[1] Ian Coddington, Nathan Newbury, and William Swann, "Dual-comb spectroscopy", Optica 3(4), 414-426 (2016).

[2] M. Vainio, L. Halonen, "Mid-infrared optical parametric oscillators and frequency combs for molecular spectroscopy", Phys. Chem. Chem. Phys, 18, 4266-4294, (2016).

[3] Z. Zhang, C. Gu, J. Sun, C.Wang, T. Gardiner and D. T. Reid, "Asynchronous midinfrared ultrafast optical parametric oscillator for dualcomb spectroscopy", Opt. Lett. 37, 187-189 (2012).

[4] Y. Jin, S.M. Cristescu, F.J.M Harren and J. Mandon, "Broadly, independent-tunable, dual-wavelength mid-infrared ultrafast optical parametric oscillator", Opt. Express 23, 20418-20427 (2015). 